\title{
Polymorphic variants of MIF gene and prognosis in steroid therapy in children with idiopathic nephrotic syndrome
}

\author{
Monika Świerczewska ${ }^{\circledR}$, Danuta Ostalska-Nowicka², Bartosz Kempisty ${ }^{1,3}$, \\ Aleksandra Szczepankiewicz ${ }^{4}$ and Michał Nowicki ${ }^{1}$
}

'Department of Histology and Embryology, Poznan University of Medical Sciences, Poland; 2Department of Pediatric Cardiology and Nephrology, Poznan University of Medical Sciences, Poland; ${ }^{3}$ Department of Anatomy, Poznan University of Medical Sciences, Poland; ${ }^{4}$ Laboratory of Molecular and Cell Biology, Department of Pulmonology, Pediatric Allergy and Clinical Immunology, Poznan University of Medical Sciences, Poland

Nephrotic syndrome (NS) is the most common reason of proteinuria in children and can be caused by the pathology of renal glomeruli. Steroid therapy is typically used in this disorder. It has been shown that MIF is a cytokine which counteracts the immunosuppressive properties of glucocorticoids. The aim of this study was looking for a correlation between MIF polymorphisms and genetic susceptibility to steroid resistance in children with INS (Idiopathic NS). Methods: The study was performed in 71 patients with INS including SRNS (steroid resistance nephrotic syndrome) (41) and SSNS (steroid sensitive nephrotic syndrome) (30) and in 30 control subjects. We employed Sanger sequencing and capillary electrophoresis. Linkage disequilibrium was made using Haploview and PHASE. Results: We didn't observe a statistical significance between SNPs detected in patients with INS and controls. Our studies revealed statistical significance for two polymorphisms: rs2070767C >T and rs2000466T>G between patients with SRNS and SSNS. The results for rs34383331T>A are close to being statistically significant. Statistical significance was revealed for CATT5/CATT6 genotype in SRNS group vs SSNS group (OR=4.604, $95 \% \mathrm{Cl}=1.356-15.632, \mathrm{p}=0.0168)$. We found that the frequency of 5/X-CATT genotype compared with $\mathrm{X} / \mathrm{X}$-CATT genotype was significantly higher in SRNS patients vs SSNS $(\mathrm{OR}=3.167,95 \% \mathrm{Cl}=1.046-9.585$, $p=0.0426)$. In linkage disequilibrium analysis we didn't show involvement in susceptibility to INS and steroid sensitive phenotype. Conclusions: Our results suggest that the role of MIF polymorphisms in the susceptibility to positive response to steroid therapy is still unresolved. It indicates that MIF may be involved in indirect and complex molecular mechanisms of steroid activity in hormone-dependent metabolic pathways in children with INS. Because of ambiguous findings, pleiotropic features of this cytokine require that more research should be undertaken.

Key words: nephrotic syndrome, steroid resistance, MIF gene, single nucleotide polymorphism, short tandem repeat

Received: 03 June, 2013; revised: 25 November, 2013; accepted: 22 January, 2014; available on-line: 18 March, 2014

\section{INTRODUCTION}

Nephrotic syndrome (NS) is the most common reason of proteinuria (more than $50 \mathrm{mg} / \mathrm{kg} /$ day or protein/ creatinine ratio $>200 \mathrm{mg} / \mathrm{mmol}$ ) in children and can be caused by the pathology of renal glomeruli (Van Hausen \& Kemper, 2011; Abid et al., 2012). The most common proteinuria in children can be associated with the idiopathic nephrotic syndrome (INS), which includes several histological variants: minimal change nephrotic syndrome (MCNS), (80\%), diffuse mesangial proliferation (DMP) and focal segmental glomerulosclerosis (FSGS) (Banaszak et al., 2011; Harambat et al., 2011). Since the 1950 s steroid treatment is the most frequently used therapy of INS. (Hodson et al., 2000). However, not all patients show positive response to the therapy and approximately $10-20 \%$ of patients don't respond to it. The variety of reactions to this therapy divides the patients to steroid sensitive (SS) and steroid resistant (SR) (Mehls \& Hoyer, 2011; Abid et al., 2012).

Actions of glucocorticoids (GCs) include the genomic and nongenomic mechanisms. Genomic reactions of GCs are associated with glucocorticoid receptor $\alpha$ (GR) (Gross et al., 2009; Mehls \& Hoyer, 2011). Nongenomic mechanisms include interactions of GCs with cell membrane, cytosolic GR and with cell membrane-bound GR (Smoak \& Cidlowski, 2004; Stahn \& Buttgereit, 2008; Alangari, 2010).

MIF (macrophage migration inhibitory factor) is a pleiotropic proinflammatory cytokine produced by many cell types such as: $\mathrm{T}$ lymphocytes, monocytes/macrophages, vascular endothelia. It is also released from the pituitary which suggests that MIF is also an endocrine factor (Berdeli et al., 2005; Gómez et al., 2007), (Fig. 1). Because of its widespread properties it is a crucial mediator of many immune and autoimmune diseases such as: juvenile idiopathic arthritis (JIA), Crohn disease, diabetes type 1, glomerulonephritis, septic shock, inflammatory lung disease and cancer (Berdeli et al., 2005; StosicGrujicic et al., 2009). Although most proinflammatory cytokines are inhibited by GCs, it was shown that MIF is released from macrophages by very low concentrations of GCs (dexamethasone or hydrocortisone at $10^{-14} \mathrm{M}$ ) in a rodent cell line (Donn \& Ray, 2004; Barnes \& Adcock, 2009). Therefore, it may suggest that MIF is a factor which suppresses the efficiency of steroid treatment (Stosic-Grujicic et al., 2009). It is assumed that many polymorphisms in the MIF genes associated with immune

e-mail: m_swierczewska@wp.p

Abbreviations: INS idiopathic nephrotic syndrome; GCs glucocorticoids; GR glucocorticoid receptor; MIF macrophage migration inhibitory factor; SSNS steroid sensitive nephrotic syndrome; SRNS steroid resistance nephrotic syndrome; SNP single nucleotide polymorphisms; STR short tandem repeat; LD linkage disequilibrium; RA rheumatoid arthritis 


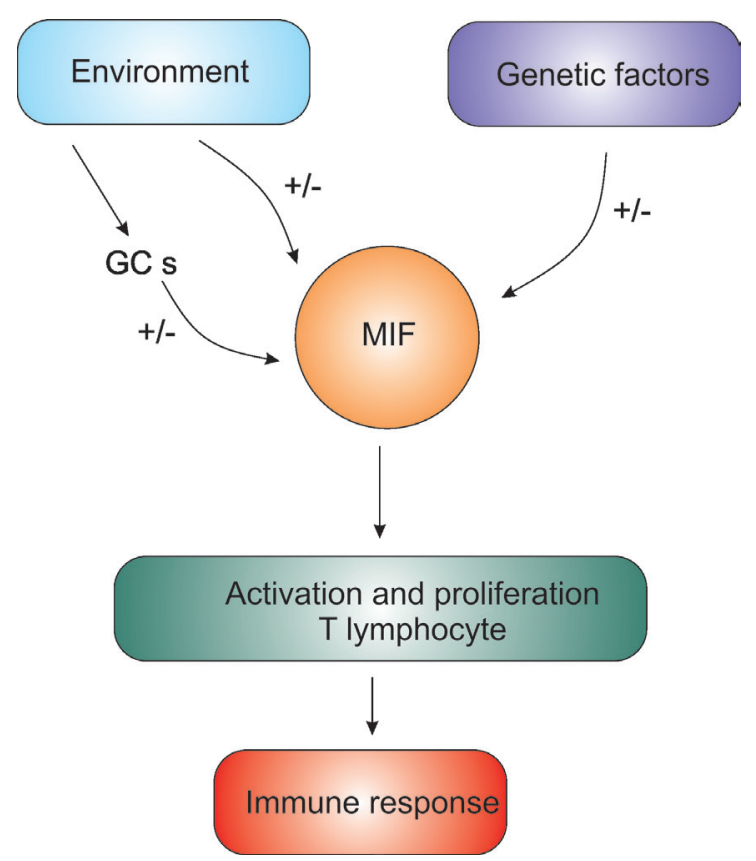

Figure 1. MIF - a mediator of the immune response.

response may influence many immune and autoimmune diseases and have a prognostic value.

The aim of this study was to analyze an association between the discovered MIF polymorphisms and glucocorticoid resistance of children with idiopathic nephrotic syndrome in the Polish population, by sequencing all of the exons in the MIF gene, with about 100bp of the neighboring intronic sequences and the 5, 3' flanking DNA regions, as well as the promoter DNA.

\section{MATERIALS AND METHODS}

Patients and controls. Seventy one (29 girls and 42 boys) patients at the ages from 3 to 19 years old (average age - 10.1 \pm 4.4 ) that were hospitalized because of idiopathic nephrotic syndrome in the years 2008-2010 at the Department of Cardiology and Children Nephrology at the Medical University of Poznan were the subject of this study. Based on steroid treatment during the induction treatment, thirty patients (13 girls and 17 boys) that had the steroid sensitive nephrotic syndrome (SSNS) and forty one children (16 girls and 25 boys) with steroid resistance nephrotic syndrome (SRNS) were selected. Histopathological classification of kidney biopsies of the SRNS children was performed by an experienced pathologist. They were also correlated with age and gender (Table 1). Thirty patients were included as controls. The mean age of the control subjects was 10.1 \pm 4.4 . Protocol of the study was approved by the Local Ethical Committee of Poznan University of Medical Sciences. Written agreement was obtained from patients and controls. All participating subjects were of Polish origin.

Genotyping. DNA isolation and PCR assay. Genomic DNA was extracted from $200 \mu$ of peripheral leukocytes using a QIA Amp DNA Blood Mini Kit (Qiagen, Hilden, Germany). The DNA was precipitated and resuspended in $200 \mu \mathrm{l}$ of the AE buffer and stored at $-20^{\circ} \mathrm{C}$ until needed.

The following sequences obtained from GenBank were used as reference sequences of MIF (Gene ID:4282): NG_012099.1: 5001-5845 (genomic). Each of
Table 1. Histopathological classification and correlation with age and gender.

SRNS

\begin{tabular}{lllll}
\hline \multirow{2}{*}{ Histopathology } & \multicolumn{3}{l}{ Age of illness onset } & Gender \\
\cline { 2 - 5 } & $\begin{array}{l}<6 \text { years } \\
\text { old }\end{array}$ & $\begin{array}{l}>6 \text { years } \\
\text { old }\end{array}$ & Female & Male \\
\hline MCD $(n=3) 7.3 \%$ & 1 & 2 & 3 & - \\
\hline FSGS $(n=7) 17.1 \%$ & 7 & - & 4 & 3 \\
\hline MP $(n=12) 29.3 \%$ & 7 & 5 & 2 & 10 \\
\hline MCD, N $(n=2) 4.9 \%$ & 2 & - & 0 & 2 \\
\hline MP, N $(n=1) 2.4 \%$ & 1 & - & 1 & - \\
\hline MP, GS $(n=1) 2.4 \%$ & 1 & - & - & 1 \\
\hline MPGM $(n=1) 2.4 \%$ & - & 1 & 1 & - \\
\hline DMP $(n=1) 2.4 \%$ & 1 & - & 1 & - \\
\hline
\end{tabular}

$M C D$, minimal change disease; FSGS, focal segmental glomerulosclerosis; MP, mesangial proliferation; N, nephritis; GS, glomerulosclerosis; MPGM, membrane proliferative; DMP, diffuse mesangial proliferation

the MIF exons, about 100bp of the neighboring intronic sequences and the 5', 3' flanking DNA regions, as well as the promoter region were amplified by PCR. PCR primers were designed using Primer 3 Input (version 0.4.0.). The PCR reaction consisted of nuclease-free water, PCR Buffer $+\mathrm{MgCl}_{2} 10 \mathrm{X}$, d NTP mix $5 \mathrm{mM}$, primer $\operatorname{mix}(\mathrm{F}+\mathrm{R}) 12.5 \mu \mathrm{M}$, GC Rich Solution 5X, FastStart Taq DNA Polymerase, genomic DNA. The PCR conditions were as follows: $95^{\circ} \mathrm{C} / 4 \mathrm{~min}$, followed by 40 cycles of: $95^{\circ} \mathrm{C} / 30 \mathrm{sec}$, annealing temperature depending on the primer, $72^{\circ} \mathrm{C} / 1 \mathrm{~min}$, and then $72^{\circ} / 7 \mathrm{~min}$. The PCR products were resolved using a $1.5 \%$ agarose gel stained with the ethidium bromide.

DNA fragment length analysis. Because of Short Tandem Polymorphism (STR) in the promoter region, the forward primer created for this region was labeled fluorescently with FAM-6 dye at the 5' end. To estimate the length of the resulting reaction products, capillary electrophoresis was carried out on Genetic Analyzer 3110 (Applied Biosystems) in the presence of GeneScan 600 LIZ marker. The results were analyzed using Peak Scanner ${ }^{\mathrm{TM}}$ software (Applied Biosystems).

DNA sequencing. PCR products were sequenced using the Big Dye ${ }^{\mathrm{TM}}$ Terminator Cycle Sequencing Kit 3.1 and AmpliTaq ${ }^{\circledR}$ polymerase (Applied Biosystems). Detection and sequencing were carried out with a 3130 ABI Genetic Analyzer (PE Applayed Biosystems). Results were analyzed using Sequencing Analysis 5.2. The data were screened for genetic variations in the MIF gene using FASTA Sequence Comparison at the U. of Virginia (www.fasta.bioch.virginia.edu) and the GenBank entry NM_002415 as the reference sequence from the National Center for Biotechnology Information SNP database (dbSNP): www.ncbi,nlm.nih.gov/SNP (accesses on September 15, 2012). The SNP data for each sample were used to calculate study-specific SNP genomic and allelic frequencies.

Statistical analysis. The Hardy-Weinberg equilibrium (HWE) assumption was assessed for both the patient and control groups by comparing the observed numbers of each genotype with those expected under the HWE for the estimated allele frequency. Moreover, the Odds Ratio (OR) and 95\% Confidence Intervals (95\% $\mathrm{CI})$ were calculated for the association between the alleles and genotypes and the risk of INS or initial steroid 
Table 2. Genotype distribution and allele frequencies of MIF polymorphisms for children with INS including SRNS and SSNS and controls.

\begin{tabular}{|c|c|c|c|c|c|c|c|c|c|c|c|}
\hline MIF & Position & $\mathrm{n}$ & Genotypes & & & $p$ & OR(95\%Cl) & Alleles & & $p$ & $\mathrm{OR}(95 \% \mathrm{Cl})$ \\
\hline rs755622 & -173 & & $\mathrm{G} / \mathrm{G}$ & $\mathrm{G} / \mathrm{C}$ & $\mathrm{C} / \mathrm{C}$ & & & G & C & & \\
\hline \multirow{4}{*}{$\mathrm{G}>\mathrm{C}$} & Controls & 30 & $\begin{array}{c}16 \\
(53.3 \%)\end{array}$ & $\begin{array}{c}13 \\
(43.3 \%)\end{array}$ & $\begin{array}{c}1 \\
(3.3 \%)\end{array}$ & \multirow{2}{*}{0.495} & \multirow{2}{*}{$\begin{array}{c}0.687 \\
(0.283-1.666) \\
\text { GC vs GG }\end{array}$} & $\begin{array}{c}45 \\
(75.0 \%)\end{array}$ & $\begin{array}{c}15 \\
(25.0 \%)\end{array}$ & \multirow{2}{*}{0.718} & \multirow{2}{*}{$\begin{array}{c}0.873 \\
(0.431-1.765)\end{array}$} \\
\hline & INS & 71 & $\begin{array}{c}43 \\
(60.6 \%)\end{array}$ & $\begin{array}{c}24 \\
(33.8 \%)\end{array}$ & $\begin{array}{c}4 \\
(5.6 \%)\end{array}$ & & & $\begin{array}{c}110 \\
(77.5 \%)\end{array}$ & $\begin{array}{c}32 \\
(22.5 \%)\end{array}$ & & \\
\hline & SSNS & 30 & $\begin{array}{c}15 \\
(50.0 \%)\end{array}$ & $\begin{array}{c}13 \\
(43.3 \%)\end{array}$ & $\begin{array}{c}2 \\
(6.7 \%)\end{array}$ & \multirow{2}{*}{0.196} & \multirow{2}{*}{$\begin{array}{c}0.453 \\
(0.164-1.256) \\
\text { GC vs GG }\end{array}$} & $\begin{array}{c}43 \\
(71.7 \%)\end{array}$ & $\begin{array}{c}17 \\
(28.3 \%)\end{array}$ & \multirow{2}{*}{0.222} & \multirow{2}{*}{$\begin{array}{c}0.566 \\
(0.256-1.251)\end{array}$} \\
\hline & SRNS & 41 & $\begin{array}{c}28 \\
(68.3 \%)\end{array}$ & $\begin{array}{c}11 \\
(26.8 \%)\end{array}$ & $\begin{array}{c}2 \\
(4.9 \%)\end{array}$ & & & $\begin{array}{c}67 \\
(81.7 \%)\end{array}$ & $\begin{array}{c}15 \\
(18.3 \%)\end{array}$ & & \\
\hline rs112568463 & -74 & & $\mathrm{~A} / \mathrm{A}$ & $\mathrm{A} / \mathrm{T}$ & $\mathrm{T} / \mathrm{T}$ & & & A & $\mathrm{T}$ & & \\
\hline \multirow{4}{*}{$A>T$} & Controls & 30 & $\begin{array}{c}27 \\
(90.0 \%)\end{array}$ & $\begin{array}{c}3 \\
(10.0 \%)\end{array}$ & $\begin{array}{c}0 \\
(0.0 \%)\end{array}$ & \multirow{2}{*}{0.358} & \multirow{2}{*}{$\begin{array}{c}0.397 \\
(0.075-2.091) \\
\text { AT vs AA }\end{array}$} & $\begin{array}{c}57 \\
(95.0 \%)\end{array}$ & $\begin{array}{c}3 \\
(5.0 \%)\end{array}$ & \multirow{2}{*}{0.365} & \multirow{2}{*}{$\begin{array}{c}0.410 \\
(0.080-2.092)\end{array}$} \\
\hline & INS & 71 & $\begin{array}{c}68 \\
(95.8 \%)\end{array}$ & $\begin{array}{c}3 \\
(4.2 \%)\end{array}$ & $\begin{array}{c}0 \\
(0.0 \%)\end{array}$ & & & $\begin{array}{c}139 \\
(97.9 \%)\end{array}$ & $\begin{array}{c}3 \\
(2.1 \%)\end{array}$ & & \\
\hline & SSNS & 30 & $\begin{array}{c}27 \\
(90.0 \%)\end{array}$ & $\begin{array}{c}3 \\
(10.0 \%)\end{array}$ & $\begin{array}{c}0 \\
(0.0 \%)\end{array}$ & \multirow{2}{*}{-} & \multirow{2}{*}{-} & $\begin{array}{c}57 \\
(95.0 \%)\end{array}$ & $\begin{array}{c}3 \\
(5.0 \%)\end{array}$ & \multirow{2}{*}{-} & \multirow{2}{*}{-} \\
\hline & SRNS & 41 & $\begin{array}{c}41 \\
(100 \%)\end{array}$ & $\begin{array}{c}0 \\
(0.0 \%)\end{array}$ & $\begin{array}{c}0 \\
(0.0 \%)\end{array}$ & & & $\begin{array}{c}82 \\
(100 \%)\end{array}$ & $\begin{array}{c}0 \\
(0.0 \%)\end{array}$ & & \\
\hline rs2096525 & $\begin{array}{l}\text { Intron } 1 \\
(255)\end{array}$ & & $\mathrm{T} / \mathrm{T}$ & $\mathrm{T} / \mathrm{C}$ & $\mathrm{C} / \mathrm{C}$ & & & $\mathrm{T}$ & C & & \\
\hline \multirow{4}{*}{$\mathrm{T}>\mathrm{C}$} & Controls & 30 & $\begin{array}{c}16 \\
(53.3 \%)\end{array}$ & $\begin{array}{c}13 \\
(43.3 \%)\end{array}$ & $\begin{array}{c}1 \\
(3.3 \%)\end{array}$ & \multirow{2}{*}{0.364} & \multirow{2}{*}{$\begin{array}{c}0.398 \\
(0.142-1.118) \\
\text { TC vs TT }\end{array}$} & $\begin{array}{c}45 \\
(75.0 \%)\end{array}$ & $\begin{array}{c}15 \\
(25.0 \%)\end{array}$ & \multirow{2}{*}{0.714} & \multirow{2}{*}{$\begin{array}{c}0.838 \\
(0.413-1.699)\end{array}$} \\
\hline & INS & 71 & $\begin{array}{c}44 \\
(62.0 \%)\end{array}$ & $\begin{array}{c}23 \\
(32.4 \%)\end{array}$ & $\begin{array}{c}4 \\
(5.6 \%)\end{array}$ & & & $\begin{array}{c}111 \\
(78.2 \%)\end{array}$ & $\begin{array}{c}31 \\
(21.8 \%)\end{array}$ & & \\
\hline & SSNS & 30 & $\begin{array}{c}15 \\
(50.0 \%)\end{array}$ & $\begin{array}{c}13 \\
(43.3 \%)\end{array}$ & $\begin{array}{c}2 \\
(6.7 \%)\end{array}$ & \multirow{2}{*}{0.117} & \multirow{2}{*}{$\begin{array}{c}0.398 \\
(0.142-1.118) \\
\text { TC vs TT }\end{array}$} & $\begin{array}{c}43 \\
(71.7 \%)\end{array}$ & $\begin{array}{c}17 \\
(28.3 \%)\end{array}$ & \multirow{2}{*}{0.150} & \multirow{2}{*}{$\begin{array}{c}0.521 \\
(0.233-1.164)\end{array}$} \\
\hline & SRNS & 41 & $\begin{array}{c}29 \\
(70.7 \%)\end{array}$ & $\begin{array}{c}10 \\
(24.4 \%)\end{array}$ & $\begin{array}{c}2 \\
(4.9 \%)\end{array}$ & & & $\begin{array}{c}68 \\
(82.9 \%)\end{array}$ & $\begin{array}{c}14 \\
(17.1 \%)\end{array}$ & & \\
\hline rs2070766 & $\begin{array}{l}\text { Intron } 2 \\
(657)\end{array}$ & & $\mathrm{C} / \mathrm{C}$ & $\mathrm{C} / \mathrm{G}$ & $\mathrm{G} / \mathrm{G}$ & & & $\mathrm{C}$ & $\mathrm{G}$ & & \\
\hline \multirow{4}{*}{$C>G$} & Controls & 30 & $\begin{array}{c}16 \\
(53.3 \%)\end{array}$ & $\begin{array}{c}12 \\
(40.0 \%)\end{array}$ & $\begin{array}{c}2 \\
(6.7 \%)\end{array}$ & 0516 & $\begin{array}{c}0.744 \\
(0.315-1.760)\end{array}$ & $\begin{array}{c}44 \\
(73.0 \%)\end{array}$ & $\begin{array}{c}16 \\
(27.0 \%)\end{array}$ & 0589 & 0.800 \\
\hline & INS & 71 & $\begin{array}{c}43 \\
(60.6 \%)\end{array}$ & $\begin{array}{c}24 \\
(33.8 \%)\end{array}$ & $\begin{array}{c}4 \\
(5.6 \%)\end{array}$ & 0.J & $\begin{array}{c}\text { CG+GG vs } \\
\text { CC }\end{array}$ & $\begin{array}{c}110 \\
(77.5 \%)\end{array}$ & $\begin{array}{c}32 \\
(22.5 \%)\end{array}$ & 0.589 & $(0.399-1.602)$ \\
\hline & SSNS & 30 & $\begin{array}{c}15 \\
(50.0 \%)\end{array}$ & $\begin{array}{c}13 \\
(43.3 \%)\end{array}$ & $\begin{array}{c}2 \\
(6.7 \%)\end{array}$ & 0.145 & $\begin{array}{c}0.464 \\
(0.176-1.227)\end{array}$ & $\begin{array}{c}43 \\
(71.7 \%)\end{array}$ & $\begin{array}{c}17 \\
(28.3 \%)\end{array}$ & 0227 & 0.566 \\
\hline & SRNS & 41 & $\begin{array}{c}28 \\
(68.3 \%)\end{array}$ & $\begin{array}{c}11 \\
(26.8 \%)\end{array}$ & $\begin{array}{c}2 \\
(4.9 \%)\end{array}$ & $0.14 J$ & $\begin{array}{c}\text { CG+GG vs } \\
\text { CC }\end{array}$ & $\begin{array}{c}67 \\
(81.7 \%)\end{array}$ & $\begin{array}{c}15 \\
(18.3 \%)\end{array}$ & 0.222 & $(0.256-1.251)$ \\
\hline rs2070767 & $\begin{array}{l}\text { 3'UTR } \\
(899)\end{array}$ & & $\mathrm{C} / \mathrm{C}$ & $\mathrm{C} / \mathrm{T}$ & $\mathrm{T} / \mathrm{T}$ & & & $\mathrm{C}$ & $\mathrm{T}$ & & \\
\hline & Controls & 30 & $\begin{array}{c}18 \\
(60.0 \%)\end{array}$ & $\begin{array}{c}10 \\
(33.0 \%)\end{array}$ & $\begin{array}{c}2 \\
(7.0 \%)\end{array}$ & 0644 & $\begin{array}{c}0.659 \\
(0101-4286)\end{array}$ & $\begin{array}{c}46 \\
(77.0 \%)\end{array}$ & $\begin{array}{c}14 \\
(23.0 \%)\end{array}$ & 1000 & 0.995 \\
\hline$C>T$ & INS & 71 & $\begin{array}{c}41 \\
(57.7 \%)\end{array}$ & $\begin{array}{c}27 \\
(38.1 \%)\end{array}$ & $\begin{array}{c}3 \\
(4.2 \%)\end{array}$ & דדרט & TT vs CC & $\begin{array}{c}109 \\
(76.8 \%)\end{array}$ & $\begin{array}{c}33 \\
(23.2 \%)\end{array}$ & 1.000 & $(0.487-2.031)$ \\
\hline & SSNS & 30 & $\begin{array}{c}21 \\
(70.0 \%)\end{array}$ & $\begin{array}{c}7 \\
(23.0 \%)\end{array}$ & $\begin{array}{c}2 \\
(7.0 \%)\end{array}$ & 0,047 & $\begin{array}{c}3.00 \\
(1043-8627)\end{array}$ & $\begin{array}{c}49 \\
(82.0 \%)\end{array}$ & $\begin{array}{c}11 \\
(18.0 \%)\end{array}$ & 0315 & 1.633 \\
\hline & SRNS & 41 & $\begin{array}{c}20 \\
(73.0 \%)\end{array}$ & $\begin{array}{c}20 \\
(49.0 \%)\end{array}$ & $\begin{array}{c}1 \\
(2.0 \%)\end{array}$ & & CT vs CC & $\begin{array}{c}60 \\
(73.0 \%)\end{array}$ & $\begin{array}{c}22 \\
(27.0 \%)\end{array}$ & נוס. & $(0.722-3.695)$ \\
\hline rs2000466 & $\begin{array}{l}\text { 3'UTR } \\
\text { (1298) }\end{array}$ & & $\mathrm{T} / \mathrm{T}$ & $\mathrm{T} / \mathrm{G}$ & $\mathrm{G} / \mathrm{G}$ & & & $\mathrm{T}$ & G & & \\
\hline & Controls & 30 & $\begin{array}{c}17 \\
(56.7 \%)\end{array}$ & $\begin{array}{c}12 \\
(40.0 \%)\end{array}$ & $\begin{array}{c}1 \\
(3.3 \%)\end{array}$ & 0660 & $\begin{array}{c}0.802 \\
(0.337-1.909)\end{array}$ & $\begin{array}{c}46 \\
(76.7 \%)\end{array}$ & $\begin{array}{c}14 \\
(23.3 \%)\end{array}$ & 0851 & 0.918 \\
\hline$G$ & INS & 71 & $\begin{array}{c}44 \\
(62.0 \%)\end{array}$ & $\begin{array}{c}23 \\
(32.4 \%)\end{array}$ & $\begin{array}{c}4 \\
(5.6 \%)\end{array}$ & 0.000 & $\begin{array}{c}\text { TG+GG vs } \\
T ा\end{array}$ & $\begin{array}{c}111 \\
(78.2 \%)\end{array}$ & $\begin{array}{c}31 \\
(21.8 \%)\end{array}$ & 0.854 & $(0.447-1.882)$ \\
\hline 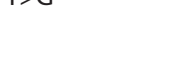 & SSNS & 30 & $\begin{array}{c}14 \\
(46.7 \%)\end{array}$ & $\begin{array}{c}14 \\
(46.7 \%)\end{array}$ & $\begin{array}{c}2 \\
(6.7 \%)\end{array}$ & ברחת & $\begin{array}{c}0.321 \\
(0.119-0.869)\end{array}$ & $\begin{array}{c}42 \\
(70.0 \%)\end{array}$ & $\begin{array}{c}18 \\
(30.0 \%)\end{array}$ & & 0.440 \\
\hline & SRNS & 41 & $\begin{array}{c}30 \\
(73.2 \%)\end{array}$ & $\begin{array}{c}9 \\
(22.0 \%)\end{array}$ & $\begin{array}{c}2 \\
(4.9 \%)\end{array}$ & 0.028 & $\begin{array}{c}\mathrm{TG}+\mathrm{GG} v s \\
T \mathrm{~T}\end{array}$ & $\begin{array}{c}69 \\
(84.1 \%)\end{array}$ & $\begin{array}{c}13 \\
(15.9 \%)\end{array}$ & 033 & $(0.196-0.989)$ \\
\hline
\end{tabular}




\begin{tabular}{|c|c|c|c|c|c|c|c|c|c|c|c|}
\hline rs34383331 & $\begin{array}{l}3^{\prime} \text { UTR } \\
(1515)\end{array}$ & & $\mathrm{T} / \mathrm{T}$ & T/A & $\mathrm{A} / \mathrm{A}$ & & & $\mathrm{T}$ & A & & \\
\hline \multirow{4}{*}{$\mathrm{T}>\mathrm{A}$} & Controls & 30 & $\begin{array}{c}17 \\
(57.0 \%)\end{array}$ & $\begin{array}{c}12 \\
(40.0 \%)\end{array}$ & $\begin{array}{c}1 \\
(3.0 \%)\end{array}$ & \multirow{2}{*}{0.650} & \multirow{2}{*}{$\begin{array}{c}0.791 \\
(0.324-1.929) \\
\text { TA vs TT }\end{array}$} & $\begin{array}{c}46 \\
(77.0 \%)\end{array}$ & $\begin{array}{c}14 \\
(23.0 \%)\end{array}$ & \multirow{2}{*}{1.000} & \multirow{2}{*}{$\begin{array}{c}0.956 \\
(0.467-1.956)\end{array}$} \\
\hline & INS & 71 & $\begin{array}{c}43 \\
(60.6 \%)\end{array}$ & $\begin{array}{c}24 \\
(33.8 \%)\end{array}$ & $\begin{array}{c}4 \\
(5.6 \%)\end{array}$ & & & $\begin{array}{c}110 \\
(77.5 \%)\end{array}$ & $\begin{array}{c}32 \\
(22.5 \%)\end{array}$ & & \\
\hline & SSNS & 30 & $\begin{array}{c}14 \\
(47.0 \%)\end{array}$ & $\begin{array}{c}14 \\
(47.0 \%)\end{array}$ & $\begin{array}{c}2 \\
(7.0 \%)\end{array}$ & \multirow{2}{*}{0.0514} & \multirow{2}{*}{$\begin{array}{c}0.362 \\
(0.135-0.968) \\
\text { TA+AA vs TT }\end{array}$} & $\begin{array}{c}42 \\
(70.0 \%)\end{array}$ & $\begin{array}{c}18 \\
(30.0 \%)\end{array}$ & \multirow{2}{*}{0.103} & \multirow{2}{*}{$\begin{array}{c}0.480 \\
(0.216-1.066)\end{array}$} \\
\hline & SRNS & 41 & $\begin{array}{c}29 \\
(71.0 \%)\end{array}$ & $\begin{array}{c}10 \\
(24.0 \%)\end{array}$ & $\begin{array}{c}2 \\
(5.0 \%)\end{array}$ & & & $\begin{array}{c}68 \\
(83.0 \%)\end{array}$ & $\begin{array}{c}14 \\
(17.0 \%)\end{array}$ & & \\
\hline
\end{tabular}

responsiveness using Fisher's exact test. The $\mathrm{p}$ value of $\leq 0.05$ was considered to indicate statistical significance. Linkage disequilibrium (LD) between SNPs polymorphisms of MIF gene was examined by pair-wise comparisons of D' using Haploview version 4.1. Where necessary, correction for multiple testing was done. Haplotype frequencies between SNP and STR polymorphisms of the MIF gene in the promoter region were calculated using the PHASE version 2.1.

\section{RESULTS}

\section{Single Nucleotide Polymorphisms.}

Seventy one patients with INS, including SRNS and SSNS, and thirty controls were genotyped. The genotypic and allelic frequencies for SNP and STR are shown in Table 2 and in Table 3. The frequency of all geno- types studied did not exhibit deviation from the HWE between all investigated groups ( $\mathrm{p}>0.05)$.

As shown in Table 2, we didn't observe a statistical significance between the detected SNPs in patients with INS and controls. Our studies revealed statistical significance for two polymorphisms: rs2070767C > T and rs2000466T $>\mathrm{G}$ between patients with SRNS and SSNS. The results for $\mathrm{rs} 34383331 \mathrm{~T}>\mathrm{A}$ are close to being statistically significant. We found that CT genotype of $\mathrm{rs} 2070767 \mathrm{C}>\mathrm{T}$ is associated with the risk of SRNS vs SSNS. The OR for SRNS children with the CT genotype compared with CC genotype was 3.00 (95\% CI=1.043$8.627, \mathrm{p}=0.047)$. In addition, we evaluated a correlation of $\mathrm{rs} 2000466 \mathrm{~T}>\mathrm{G}$ with $\mathrm{SRNS}$ vs SSNS (OR $=0.321$, $95 \% \mathrm{CI}=0.119-0.869, \mathrm{p}=0.028)$. The distribution of $\mathrm{TG}$ genotype is about 2-fold higher in SSNS children than in SRNS (46.7\% and $22.0 \%$, respectively), and the occurrence of the GG genotype is about 1.5-fold higher in SSNS children than in SRNS $(6.7 \%$ and $4.9 \%$, respective-

Table 3. Data from MIF -794 (CATT)5-8 microsatellite (rs5844572) in patients with INS including SRNS and SSNS and controls.

\begin{tabular}{|c|c|c|c|c|c|}
\hline Polymorphisms & Genotypes & INS n=71 (\%) & Controls n=30 (\%) & OR $(95 \% \mathrm{Cl})$ & $p$ value \\
\hline rs5844572 & $5 / 5$ & $6(8.45 \%)$ & $2(6.67 \%)$ & $1.292(0.246-6.800)$ & 1.00 \\
\hline \multirow[t]{20}{*}{ САТТ 5-7 } & $5 / 6$ & $21(29.58 \%)$ & $8(26.67 \%)$ & $1.155(0.444-3.006)$ & 0.815 \\
\hline & $5 / 7$ & $4(5.63 \%)$ & $2(6.67 \%)$ & $0.836(0.145-4.828)$ & 1.00 \\
\hline & $6 / 6$ & $25(35.21 \%)$ & $8(26.67 \%)$ & $1.495(0.581-3.843)$ & 0.490 \\
\hline & $6 / 7$ & $13(18.31 \%)$ & $10(33.33 \%)$ & $0.448(0.170-1.181)$ & 0.122 \\
\hline & $7 / 7$ & $2(2.82 \%)$ & $0(0 \%)$ & - & 1.00 \\
\hline & Alleles & & & & \\
\hline & 5 & $37(26.06 \%)$ & $14(23.33 \%)$ & $1.158(0.572-2.345)$ & 0.727 \\
\hline & 6 & $84(59.15 \%)$ & $34(56.67 \%)$ & $1.108(0.602-2.039)$ & 0.757 \\
\hline & 7 & $21(14.79 \%)$ & $12(20 \%)$ & $0.694(0.317-1.521)$ & 0.406 \\
\hline & Genotypes & SRNS n=41 (\%) & SSNS n=30 (\%) & OR $(95 \% \mathrm{Cl})$ & $p$ value \\
\hline & $5 / 5$ & $2(4.88 \%)$ & $4(13.33 \%)$ & $0.333(0.057-1.954)$ & 0.233 \\
\hline & $5 / 6$ & $17(41.46 \%)$ & $4(13.33 \%)$ & $4.604(1.356-15.632)$ & 0.017 \\
\hline & $5 / 7$ & $2(4.88 \%)$ & $2(6.67 \%)$ & $0.718(0.095-5.408)$ & 1.00 \\
\hline & $6 / 6$ & $13(31.71 \%)$ & $12(40 \%)$ & $0.696(0.621-1.861)$ & 0.616 \\
\hline & $6 / 7$ & $6(14.63 \%)$ & $7(23.33 \%)$ & $0.563(0.168-1.890)$ & 0.371 \\
\hline & $7 / 7$ & $1(2.4 \%)$ & $1(3.33 \%)$ & $0.725(0.044-12.076)$ & 1.00 \\
\hline & Alleles & & & & \\
\hline & 5 & $23(28 \%)$ & $14(23.33 \%)$ & $1.281(0.594-2.761)$ & 0.567 \\
\hline & 6 & $49(59.8 \%)$ & $35(58.33 \%)$ & $1.061(0.539-2.087)$ & 0.865 \\
\hline & 7 & $10(12.2 \%)$ & $11(18.33 \%)$ & $0.619(0.244-1.568)$ & 0.345 \\
\hline
\end{tabular}




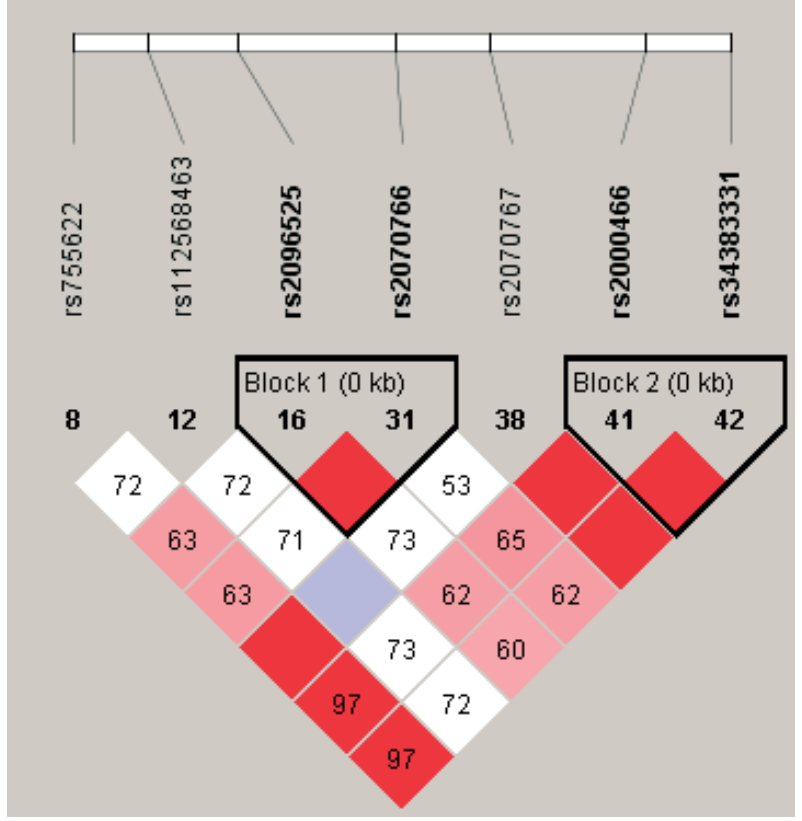

Figure. 2. Relative positions and LD estimates between 7 MIF polymorphisms in the analyzed population (controls and INS). Colored squares correspond to $D^{\prime}$ values with numerical estimates given within the squares.

ly). A similar situation was observed for rs34383331T $>$ A for SRNS vs SSNS (OR=0.362, 95\% $\mathrm{CI}=0.135-0.968$, $\mathrm{p}=0.0514$ ) (Table 2).

\section{Short Tandem Repeat in the promoter region}

The microsatellite DNA (rs5844572, CATT5-8) in the MIF gene has four CATT repeat units $(5,6,7$ and 8 CATT repeats). In our research we didn't detect any 8 CATT repeats (Table 3 ). We observed the highest distribution of the CATT5/CATT6 genotype in SRNS patients $(41.46 \%)$ and CATT6/CATT7 genotype in controls $(33.33 \%)$. Our studies revealed a statistical signifi-

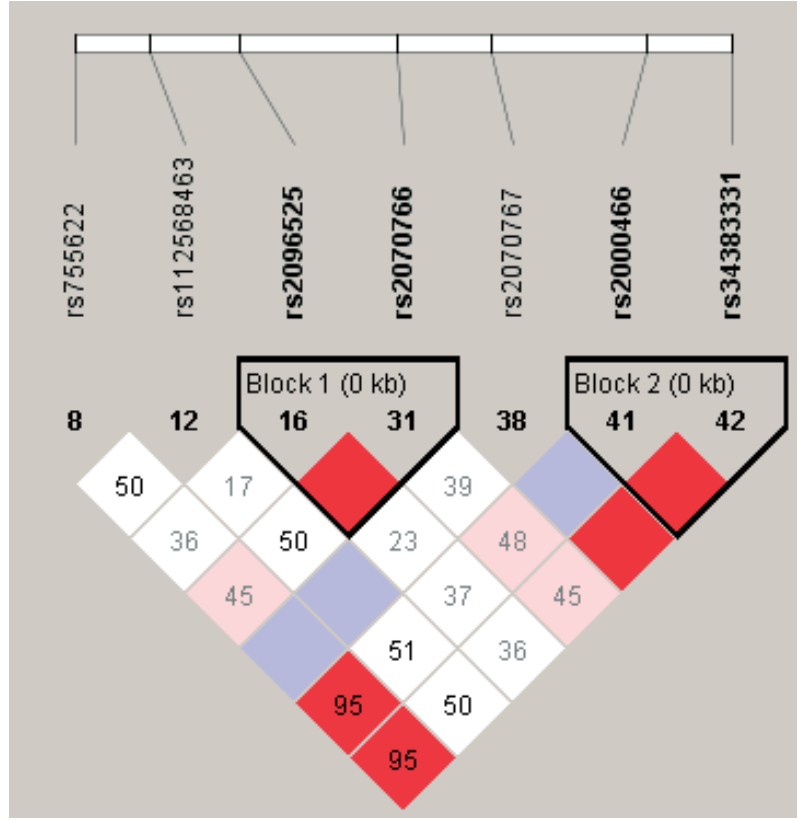

Figure. 3. Relative positions and LD estimates between 7 MIF polymorphisms in the analyzed population (SRNS and SSNS). Colored squares correspond to $D^{\prime}$ values with numerical estimates given within the squares.

cance for the genotype CATT5/CATT6 in SRNS group vs SSNS group $(\mathrm{OR}=4.604,95 \% \quad \mathrm{CI}=1.356-15.632$, $\mathrm{p}=0.0168)$. We didn't evaluate the genotype CATT7/ CATT7 in controls, whereas it was present in others (INS - 2.82\%, SRNS - 2.4\%, SSNS - 3.33\%). In addition, in Table 3 we make the association between tetranucleotide repeat polymorphism and INS and SRNS and SSNS. We found that the frequency of $5 / \mathrm{X}-\mathrm{CAT} T$ genotype compared with X/X-CATT genotype was significantly higher in SRNS patients vs SSNS (OR=3.167, $95 \% \mathrm{CI}=1.046-9.585, \mathrm{p}=0.0426)$. In addition, there were no significant differences in other groups. We also made

Table 4. The association between tetranucleotide repeat polymorphism between groups INS vs controls and SRNS vs SSNS.

\begin{tabular}{|c|c|c|c|c|c|c|c|c|}
\hline \multirow[t]{2}{*}{ Overall } & \multicolumn{3}{|c|}{ Genotype (n) } & \multirow[t]{2}{*}{ OR $(95 \% \mathrm{Cl})$} & \multicolumn{3}{|l|}{ Genotype (n) } & \multirow[t]{2}{*}{ OR $(95 \% \mathrm{Cl})$} \\
\hline & $5 / 5$ & $5 / X$ & $X / X$ & & $\mathrm{Y} / \mathrm{Y}$ & $7 / Y$ & $7 / 7$ & \\
\hline & & & & $0.333(0.057-1.954)$ & & & & $0.725(0.044-12.076)$ \\
\hline SRNS & $2(4.88 \%)$ & 19(46.34\%) & $20(48.78 \%)$ & $\mathrm{p}=0.233$ (5/5 vs others) & $32(78.05 \%)$ & $8(19.51 \%)$ & $1(2.44 \%)$ & $\mathrm{p}=1.00$ (7/7 vs others) \\
\hline \multicolumn{9}{|l|}{$\mathrm{n}=41$} \\
\hline SSNS & $4(13.33 \%)$ & $6(20 \%)$ & $20(66.67 \%)$ & $3.167(1.046-9.585)$ & $20(66.67 \%)$ & $9(30 \%)$ & $1(3.33 \%)$ & $0.556(0.184-1.676)$ \\
\hline$n=30$ & & & & \multicolumn{3}{|l|}{$p=0.0426(5 / X$ vs $X / X)$} & & $\mathrm{p}=0.397(7 / \mathrm{Y}$ vs $\mathrm{Y} / \mathrm{Y})$ \\
\hline INS & $6(8.45 \%)$ & $25(35.21 \%)$ & $40(56.34 \%)$ & $1.292(0.248-7.347)$ & $52(73.24 \%)$ & $17(23.94 \%)$ & $2(2.82 \%)$ & $0.490(0.1968-1.2218)$ \\
\hline $\mathrm{n}=71$ & & & & \multicolumn{3}{|l|}{$p=1.00(5 / 5$ vs others $)$} & & \multirow[t]{2}{*}{$\mathrm{p}=0.151(7 / \mathrm{Y}$ vs $\mathrm{Y} / \mathrm{Y})$} \\
\hline $\begin{array}{l}\text { Con- } \\
\text { trols }\end{array}$ & $2(6.67 \%)$ & $10(33.33 \%)$ & $18(60 \%)$ & & $18(60 \%)$ & $12(40 \%)$ & $0(0 \%)$ & \\
\hline \multicolumn{9}{|l|}{$\mathrm{n}=30$} \\
\hline \multicolumn{3}{|c|}{$X-6$ or 7 repeats } & $5 / 7 ; 6 / 7 ; 7 / 7$ & \multicolumn{4}{|c|}{ „high expression” genotypes } & \\
\hline \multicolumn{3}{|c|}{$Y-5$ or 6 repeats } & $5 / 5 ; 5 / 6 ; 6 / 6$ & \multicolumn{4}{|c|}{ „low expression' genotypes } & \\
\hline
\end{tabular}


Table 5. The correlation between rs755622 G>C and rs5844572(CATT5-8) in children with INS including SRNS and SSNS and controls.

\begin{tabular}{|c|c|c|c|c|}
\hline Genotypes & SRNS & SSNS & OR $(95 \% \mathrm{Cl})$ & $p$ value \\
\hline (CATT)5/5_GG & $1(2.4 \%)$ & $4(13.3 \%)$ & $0.163(0.0172-1.536)$ & 0.155 \\
\hline (CATT)5/5_GC & $1(2.4 \%)$ & $0(0 \%)$ & - & 1.00 \\
\hline (CATT)5/6_GG & $16(39 \%)$ & $2(6.7 \%)$ & 8.96(1.8719-42.888) & 0.002 \\
\hline (CATT)5/6_GC & $1(2.4 \%)$ & $2(6.7 \%)$ & $0.35(0.030-4.050)$ & 0.570 \\
\hline (CATT)5/7_GC & $2(4.9 \%)$ & $2(6.7 \%)$ & $0.718(0.095-5.408)$ & 1.00 \\
\hline (CATT)6/6_GG & $11(26.8 \%)$ & $9(30 \%)$ & $0.856(0.302-2.427)$ & 0.795 \\
\hline (CATT)6/6_GC & $2(4.9 \%)$ & $3(10 \%)$ & $0.462(0.072-2.951)$ & 0.645 \\
\hline (CATT)6/7_GC & $5(12.2 \%)$ & $6(20 \%)$ & $0.556(0.152-2.027)$ & 0.509 \\
\hline (CATT)6/7_CC & $1(2.4 \%)$ & $1(3.3 \%)$ & $0.725(0.044-12.076)$ & 1.00 \\
\hline (САTT)7/7_CC & $1(2.4 \%)$ & $1(3.3 \%)$ & $0.725(0.044-12.076)$ & 1.00 \\
\hline Genotypes & Controls & INS & OR $(95 \% \mathrm{Cl})$ & $p$ value \\
\hline (CATT)5/5_GG & $2(6.7 \%)$ & $5(7 \%)$ & $1.061(0.194-5.796)$ & 1.00 \\
\hline (CATT)5/5_GC & $0(0 \%)$ & $1(1.4 \%)$ & - & 0.307 \\
\hline (CATT)5/6_GG & $8(26.7 \%)$ & $18(25.4 \%)$ & $0.934(0.354-2.464)$ & 1.00 \\
\hline (CATT)5/6_GC & $0(0 \%)$ & $3(4.2 \%)$ & - & 0.553 \\
\hline (CATT)5/7_GC & $2(6.7 \%)$ & $4(5.6 \%)$ & $0.836(0.145-4.828)$ & 1.00 \\
\hline (CATT)6/6_GG & $6(20 \%)$ & $20(28.2 \%)$ & $1.569(0.558-4.408)$ & 0.462 \\
\hline (CATT)6/6_GC & $2(6.7 \%)$ & $5(7 \%)$ & $1.061(0.195-5.796)$ & 1.00 \\
\hline (CATT)6/7_GC & $9(30 \%)$ & $11(15.5 \%)$ & $0.428(0.156-1.176)$ & 0.108 \\
\hline (САТT)6/7_CC & $1(3.3 \%)$ & $2(2.8 \%)$ & $0.841(0.073-9.638)$ & 1.00 \\
\hline (CATT)7/7_CC & $0(0 \%)$ & $2(2.8 \%)$ & - & 1.00 \\
\hline
\end{tabular}

(CATT)5/5_GG — low risk; (CATT)7/7_CC — high risk

a correlation between two promoter polymorphisms: rs755622G $>C$ and rs5844572 (CATT5-8) (Table 5). We observed a significant association only in groups SRNS vs SSNS for CAT'T5/CATT6_GG genotypes (OR=8.960, $95 \% \mathrm{CI}=1.872-42.888, \mathrm{p}=0.002)$. The frequency of CATT5/CATT6_GG genotypes was 5.8-fold higher in the SRNS groups when compared with SSNS $(39.0 \%$ and $6.7 \%$ respectively).

\section{Linkage disequilibrium and haplotype analysis}

In haplotype blocks for patients with INS and controls, SRNS and SSNS, we observed a strong linkage disequilibrium between four of the seven MIF polymorphisms analyzed. The program revealed two blocks (Fig. 2, Table 7). Our results for patients with INS and controls showed that none of the analyzed SNPs is associated with INS $(p>0.05)$. We observed four haplotypes, two in the first block and two in the second block (estimated population frequency $>0.05$ ). The haplotype analyses for steroid resistance phenotype also revealed two blocks (Fig. 3, Table 8). We observed three haplotypes in one block and two haplotypes in the second block. We observed that two markers (rs112568463A $>\mathrm{T}$ and rs2000466 $>$ G) revealed correlation with steroid resistance phenotype $(p=0.0407$ and $\mathrm{p}=0.0438$ respectively, Table 9). In addition, we found that the GA haplotype (rs2000466T $>$ G, rs34383331T $>$ A) may be protective in regard to the steroid resistance phenotype in INS children $(p=0.0438)$, although the association was not sufficiently strong to survive adjusting the significance level for multiple comparisons using 10000 permutations. Also, Table 6 presents $M I F$ haplotypes for polymorphisms located in the promoter region: $-173 \mathrm{G} / \mathrm{C}$ (rs755622) and -794 CAT'T (rs5844572). As we can see, the $\mathrm{C}$ allele is in a high linkage disequilibrium with CATT7 allele. The $\mathrm{C}$ allele is more frequently associated with CATT7 than with CATT5 or CAT'T6 in all groups. Accordingly, the $\mathrm{G}$ allele is more frequently associated with CAT'T6 than with CATT5 or CATT7 in

Table 6. MIF Haplotype Frequencies in patients with INS including SRNS and SSNS and controls (according to PHASE).

\begin{tabular}{|c|c|c|c|c|c|c|c|c|}
\hline SRNS & & & & SSNS & & & & \\
\hline index & haplotype & $E($ freq $)$ & S.E & index & hap & type & $E$ (freq) & S.E \\
\hline 1 & $5 \mathrm{G}$ & $0.262717(26.2 \%)$ & 0.008079 & & 1 & $5 \mathrm{G}$ & $0.222951(22.2 \%)$ & 0.012248 \\
\hline 2 & $5 C$ & $0.005576(0.6 \%)$ & 0.008079 & & 2 & $5 C$ & $0.010383(1 \%)$ & 0.012248 \\
\hline 3 & $6 \mathrm{G}$ & $0.553182(55.3 \%)$ & 0.008856 & & 3 & $6 \mathrm{G}$ & $0.490779(49.1 \%)$ & 0.013661 \\
\hline 4 & $6 C$ & $0.056574(5.7 \%)$ & 0.008856 & & 4 & $6 C$ & $0.092555(9.3 \%)$ & 0.013661 \\
\hline 5 & $7 \mathrm{G}$ & $0.001174(0.1 \%)$ & 0.003632 & & 5 & $7 \mathrm{G}$ & $0.002937(0.3 \%)$ & 0.006939 \\
\hline 6 & $7 \mathrm{C}$ & 0.120777 (12.1\%) & 0.003632 & & 6 & $7 \mathrm{C}$ & $0.180396(18 \%)$ & 0.006939 \\
\hline INS & & & & Contr & & & & \\
\hline index & haplotype & $E($ freq $)$ & S.E & index & hap & type & $E($ freq) & S.E \\
\hline 1 & $5 \mathrm{G}$ & $0.249132(24.9 \%)$ & 0.006095 & & 1 & $5 \mathrm{G}$ & $0.233005(23.3 \%)$ & 0.002319 \\
\hline 2 & $5 \mathrm{C}$ & $0.004389(0.4 \%)$ & 0.006095 & & 2 & $5 \mathrm{C}$ & $0.000328(0.03 \%)$ & 0.002319 \\
\hline 3 & $6 \mathrm{G}$ & $0.525231(52.5 \%)$ & 0.006046 & & 3 & $6 \mathrm{G}$ & $0.512798(51.2 \%)$ & 0.008737 \\
\hline 4 & $6 C$ & $0.073361(7.3 \%)$ & 0.006046 & & 4 & $6 C$ & $0.053869(5.4 \%)$ & 0.008737 \\
\hline 5 & $7 \mathrm{G}$ & $0.000286(0.03 \%)$ & 0.001392 & & 5 & $7 \mathrm{G}$ & $0.004197(0.4 \%)$ & 0.009279 \\
\hline 6 & $7 \mathrm{C}$ & $0.147602(14.8 \%)$ & 0.001392 & & 6 & $7 \mathrm{C}$ & $0.195803(19.6 \%)$ & 0.009279 \\
\hline
\end{tabular}


Table 7. The value of $D^{\prime}, L O D$ and $R^{2}$.

\begin{tabular}{llllll}
\hline INS and controls & L1 & L2 & $D^{\prime}$ & LOD & $R^{2}$ \\
\hline & rs2096525 & rs2070766 & 1.0 & 32.92 & 0.946 \\
\cline { 3 - 6 } & rs2000466 & rs34383331 & 1.0 & 33.9 & 0.972 \\
\hline SRNS and SSNS & & & & & \\
\hline & rs2096525 & rs2070766 & 1.0 & 19.36 & 0.844 \\
\hline & rs2000466 & rs34383331 & 1.0 & 23.51 & 0.96 \\
\hline
\end{tabular}

all groups. Unfortunately we didn't observe any differences between the groups.

\section{DISCUSSION}

The present study was designed to investigate association between MIF cytokine polymorphisms in the Polish children from Wielkopolska with INS and their response to steroids.

MIF is a proinflammatory cytokine which plays an important role in many immune and inflammatory diseases so that it can be a good prognostic factor in many therapies (Berdeli et al., 2005). It was shown that MIF also plays an important role in kidney diseases (Lan, 2008). Moreover it counter-regulates glucocorticoids effect by suppressing their therapeutic effects (Lolis, 2001; Berdeli et al., 2005). It was found that glucocorticoids induce the synthesis of $\mathrm{I} \varkappa \mathrm{B}$, whereas MIF reduces $\mathrm{I} \varkappa \mathrm{B}$. Consequently NF- $x \mathrm{~B}$ translocates to the nucleus as a free factor where it initiates transcription of specific genes. This is one of the ways to enhance an inflammatory response. Similarly, glucocorticoids also inhibit activity of the phospholipase $\mathrm{A}_{2}\left(\mathrm{PLA}_{2}\right)$ but MIF counteracts by stimulating the ERK1/2 kinases pathways, activating PLA , releasing $^{2}$ arachidonic acid and as a consequence leukotriens and prostaglandins are expressed (Renner et al., 2005; Bucala, 2013). Several studies have reported a functional significance of the MIF gene polymorphisms. Our results show that frequency of the CT genotype rs $2070767 \mathrm{C}>\mathrm{T}$, which is located in the 3'UTR region, is significantly higher in the SRNS group than in SSNS children. We also found that other two polymorphisms located in the 3'UTR region (rs2000466'T $>\mathrm{G}$ and $\mathrm{rs} 34383331 \mathrm{~T}>\mathrm{A}$ ) are correlated with SSNS when compared with SRNS. In contrast to previous findings, however, no evidence of $\mathrm{rs} 2070767 \mathrm{C}>\mathrm{T}$, rs $2000466 \mathrm{~T}>\mathrm{G}$ and $\mathrm{rs} 34383331 \mathrm{~T}>\mathrm{A}$ were determined in patients with INS, and SSNS or SRNS. Li Gao et al. (2007) examined six polymorphisms of the MIF gene in Acute Lung Injury. They explained

Table 8. Haplotype associations between groups SRNS i SSNS

\begin{tabular}{lllll}
\hline \multicolumn{1}{l}{ SSNS i SRNS } & & & \\
\hline Haplotype & Frequency & Case, Control Frequencies & Chi Square & P Value \\
\hline Block 1 & & & & \\
\hline TC & 0.775 & $0.817,0.717$ & 2.001 & 0.1572 \\
\hdashline CG & 0.197 & $0.171,0.233$ & 0.858 & 0.3544 \\
\hdashline TG & 0.028 & $0.012,0.050$ & 1.809 & 0.1786 \\
\hline Block 2 & & & & \\
\hline TT & 0.775 & $0.829,0.700$ & 3.317 & 0.0686 \\
\hdashline GA & 0.218 & $0.159,0.300$ & 4.063 & $0.0438^{*}$ \\
\hline
\end{tabular}

the influence of polymorphisms in regulatory elements in the 3'UTR on mRNA stability as well as subcellular localization of transcripts.

Zheng X et al. (2012) examined whether polymorphisms of the MIF gene are associated with Behçet's disease (BD) in the Han Chinese population. They showed significant association of two SNPs: rs $755622 \mathrm{G}>\mathrm{C}$ and rs2096525T $>C$ with BD. Additionally, they suggested that the involvement of $M I F$ in $\mathrm{BD}$ may be through regulation of its $\mathrm{mRNA}$ expression (Zheng et al., 2012). Berdeli et al. (Berdeli et al., 2005) showed correlation of the MIF -173C allele with the INS and SRNS in children in the Turkish population. Similar results were obtained by Vivarelli et al. (2008) in respect to children from Italian population. Polymorphism rs $755622 \mathrm{G}>\mathrm{C}$ in the promoter region has been correlated with a high level of MIF protein as the transition of $G$ to $C$ creates an activator protein 4 response element (Berdeli et al., 2005, Gómez et al., 2007). However, these findings are not in the agreement with our results. This observation is in agreement with Choi's findings which showed no association between the rs755622G $>$ C MIF polymorphism and clinical parameters, renal histology and steroid responsiveness (Choi et al., 2011). It seems possible that these results are due to variations in ethnic and geographic distribution of INS. Similar results were shown for other inflammatory diseases. Berdeli et al. (2006) did not reveal the role of $-173 \mathrm{C}$ allele in susceptibility to JRA (juvenile rheumatoid arthritis) in children from the Turkish population. On the other hand, Donn et al. (2002) presented association between $-173 \mathrm{C}$ allele and JIA (juvenile idiopathic arthritis) in the UK population. Positive results were also shown in the Chinese population with RA (rheumatoid arthritis) (Liu et al., 2012; Xie et al., 2012).

Several studies have been carried out on the influence of short tandem repeat (STR) CATT5-8 (rs5844572) in the promoter region of $M I F$ and its influence on many inflammatory diseases (Renner et al., 2005; Li et al., 2012). It was shown in these studies that the number of this tetranucleotide repeat is correlated with higher MIF expression taking into account population diversity (Bucala, 2013). In our study we found that the frequency of $5 / \mathrm{X}$ CATT genotype compared with X/X-CATT genotype was significantly higher in the SRNS patients vs SSNS. In addition, we observed correlation between two promoter polymorphisms in MIF, which showed that the $\mathrm{C}$ allele is in a high linkage disequilibrium with CATT7 allele and the $G$ allele is more frequently associated with CATT6. Similar results were obtained by Sreih et al. (2011) who showed dual effect of the MIF gene according to the severity of SLE. They revealed that the presence of high expression of MIF haplotype 7C correlates with a lower form of SLE in Caucasians and lower range in African-Americans. They showed that increased MIF production due to genetic factors may correlate with protection against autoimmune response associated with SLE. According to our research it is suggested that it may be associated with a positive response in steroid therapy. In contrast to previous findings, Gázquez et al. (2012) showed no association of functional variants in the promoter region of MIF gene with disease susceptibility or hearing loss progression in patients with Méniére's disease. However, the findings 
Table 9. Single marker association between groups SRNS and SSNS

\begin{tabular}{cccccc}
\hline Name & Assoc. Allele & $\begin{array}{l}\text { SRNS, SSNS } \\
\text { Ratio Counts }\end{array}$ & $\begin{array}{l}\text { SRNS, SSNS } \\
\text { Frequencies }\end{array}$ & Chi square & P value \\
\hline rs755622 & G & $67: 15,43: 17$ & $0.817,0.717$ & 2.001 & 0.1572 \\
\hline rs112568463 & A & $82: 0,57: 3$ & $1.000,0.950$ & 4.188 & 0.0407 \\
\hline rs2096525 & T & $68: 14,46: 14$ & $0.829,0.767$ & 0.858 & 0.3544 \\
\hline rs2070766 & C & $67: 15,43: 17$ & $0.817,0.717$ & 2.001 & 0.1572 \\
\hline rs2070767 & T & $22: 60,11: 49$ & $0.268,0.183$ & 1.402 & 0.2364 \\
\hline rs2000466 & T & $69: 13,42: 18$ & $0.841,0.700$ & 4.063 & 0.0438 \\
\hline rs34383331 & T & $68: 14,42: 18$ & $0.829,0.700$ & 3.317 & 0.0686 \\
\hline
\end{tabular}

of tour study do not support the previous research in autoimmune diseases. Baugh et al. (2002) identified that the low expression of 5-CATT repeat allele was correlated with low inflammation in RA. They also analyzed rs755622G $>$ C but it wasn't associated with RA (Baugh $e t$ al., 2002). A similar situation was observed in the Japanese population with ulcerative colitis (UC). Shiroeda et al. (2010) showed that genotype 5/5-CATT was a decreased risk for UC, but additional genotype 7/7-CATT was correlated with chronic continuous phenotype and distal colitis phenotype. They didn't find association with polymorphism rs $755622 \mathrm{G}>\mathrm{C}$.

Histological correlation with age and gender showed that all cases of FSGS (Focal Segmental Glomerulosclerosis) occurred in children under 6 years old and 83.3\% cases of MP (Mesangial Proliferation) were observed in boys (Table 1). We did not find any association between histological type of glomerulonephritis and SNPs. However, we observed that the most frequent types: FSGS and MP, were weakly correlated with the decreased number of repeats in the STR polymorphism (CATT5/6, CATT6/6 and CATT5/6_GG, CATT5/6_ GG, CATT6/6_GG) (not shown).

In addition, Matsumoto et al. (2005) showed that MIF levels in the serum increased in patients with FSGS and steroid-resistance NS. However, further work is required to establish the association with INS. Moreover, according to Bucala (2013), MIF interacts with Jab1 (Jun activation domain-binding protein 1$)$, which is a co-activator of the transcription factor AP-1. AP-1 participates in transcription of many proinflammatory cytokines. The ability of MIF to switch off this pathways suggests that MIF can also play a role in anti-inflammatory mechanisms (Donn \& Ray, 2004; Bucala, 2013), which may indicate pleiotropic features of MIF.

In summary, the study presented here was designed to determine the genetic factors of INS as well as SRNS. We discovered seven SNPs polymorphisms and one STR polymorphism in the MIF gene. Our results suggest that the influence of MIF polymorphisms on positive response to the steroid therapy is rather weak. Because of ambiguous findings, pleiotropic features of this cytokine require that more research should be undertaken. In addition, most polymorphisms in our studies are in nonfunctional variants. This suggests that they may be in linkage disequilibrium with polymorphisms of other genes or because of localization in regulatory elements in the 3'UTR region, their mRNA stability might be influenced. In summary, MIF may be involved in complex and indirect molecular mechanisms of steroid resistance in children with INS. 3: 170-176.

\section{Conflict of interest}

No conflict declared.

\section{Acknowledgements}

Financial support: supported by grant No. N N401 528140 from the Polish Ministry of Science and Higher Education and NR 130033 10/2010 State Committee for Scientific Research, NCBR.

\section{REFERENCES}

Abid A, Khaliq S, Shahid S, Lanewala A, Mubarak M, Hashmi S, Kazi J, Masood T, Hafeez F, Naqvi SA, Rizvi SA, Mehdi SQ (2012) A spectrum of novel NPHS1 and NPHS2 gene mutations in pediatric nephrotic syndrome patients from Pakistan. Gene 502: 133-137.

Alangari AA (2010), Genomic and non-genomic actions of glucocorticoids in asthma. Ann Thorac Med 5: 133-139.

Banaszak B, Banaszak P, Adamczyk P, Ziora K (2011) The onset of childhood nephrotic syndrome - clinical characteristics of steroidsensitive and steroid-resistant patients. Przeglad Pediatr 41: 147-151.

Barnes PJ, Adcock IM (2009) Glucocorticoid resistance in inflammatory diseases. Lancet 373: 1905-1917.

Baugh JA, Chitnis S, Donnelly SC, Monteiro J, Lin X, Plant BJ, Wolfe F, Gregersen PK, Bucala R (2002) A functional promoter polymorphism in the macrophage migration inhibitory factor (MIF) gene associated with disease severity in rheumatoid arthritis. Genes Immun

Berdeli A, Mir S, Ozkavin N, Serdaroglu E, Tabel Y, Cura A (2005) Association of macrophage migration inhibitory factor $-173 \mathrm{C}$ allele polymorphism with steroid resistance in children with nephrotic syndrome. Pediatr Nephrol 20: 1566-1571.

Berdeli A, Ozyürek AR, Ulger Z, Gürses D, Levent E, Salar K, Gürpinar AR (2006) Association of macrophage migration inhibitory factor gene $-173 \mathrm{G} / \mathrm{C}$ polymorphism with prognosis in turkish children with juvenile rheumatoid arthritis. Rheumatol Int 26: 726-731.

Bucala R (2013) MIF, MIF Alleles, and Prospects for Therapeutic Intervention in Autoimmunity. J Clin Immunol 33: 72-78.

Choi HJ, Cho HY, Ro H, Lee SH, Han KH, Lee H, Kang HG, Ha IS, Choi Y, Cheong HI (2011) Polymorphisms of the MDR1 and MIF genes in children with nephrotic syndrome. Pediatr Nephrol 26: 1981-1988.

Donn R, Alourfi Z, De Benedetti F, Meazza C, Zeggini E, Lunt M, Stevens A, Shelley E, Lamb R, Ollier WE, Thomson W, Ray D (2002) Mutation screening of the macrophage migration inhibitory factor gene positive association of a functional polymorphism of macrophage migration inhibitory factor with juvenile idiopathic arthritis. Arthritis Rheum 46: 2402-2409.

Donn R, Ray DW (2004) Macrophage migration inhibitory factor: molecular, cellular and genetic aspects of a key neuroendocrine molecule. J Endocrinol 182: 1-9.

Gao L, Flores C, Fan-Ma S, Miller EJ, Moitra J, Moreno L, Wadgaonkar R, Simon B, Brower R, Sevransky J, Tuder RM, Maloney JP, Moss M, Shanholtz C, Yates CR, Meduri GU, Ye SQ, Barnes KC, Garcia JG (2007) Macrophage migration inhibitory factor in acute lung injury: expression, biomarker, and associations. Transl Res 150: $18-29$.

Gázquez I, Moreno A, Requena T, Ohmen J, Santos-Perez S, Aran I, Soto-Varela A, Pérez-Garrigues H, López-Nevot A, Batuecas A, Friedman RA, López-Nevot MA, López-Escamez JA (2012) Functional variants of MIF, INFG and TFNA genes are not associated with disease susceptibility or hearing loss progression in patients with Ménière's disease. Eur Arch Otorbinolaryngol 270: 1521-1529.

Grieb G, Merk M, Bernhagen J, Bucala R (2010) Macrophage migration inhibitory factor (MIF): a promising biomarker. Drug News Perspect 23: 257-264.

Gross KL, Lu NZ, Cidlowski JA (2009) Molecular mechanisms regulating glucocorticoid sensitivity and resistance. Mol Cell Endocrinol 300: 7-16.

Gómez LM, Sánchez E, Ruiz-Narvaez EA, López-Nevot MA, Anava JM, Martín J (2007) Macrophage migration inhibitory factor gene influences the risk of developing tuberculosis in northwestern Colombian population. Tissue Antigens 70: 28-33. 
Harambat J, Godron A, Ernould S, Rigothier C, Llanas B, Leroy S (2013) Prediction of steroid-sparing agent use in childhood idiopathic nephrotic syndrome. Pediatr Nephrol 28: 631-638.

Hodson EM, Knight JF, Willis NS, Craig JC (2000) Corticosteroid therapy in nephrotic syndrome: a meta-analysis of randomised controlled trials. Arch Dis Child 83: 45-51.

Lan HY, Role of Macrophage Migration Inhibition Factor in Kidney Disease (2008). Nephron Exp Nephrol 109: e79-e83.

Li Y, Zeng Z, Deng S (2012) Study of the relationship between human MIF level, MIF-794CATT5-8 microsatellite polymorphism, and susceptibility of tuberculosis in Southwest China. Braz J Infect Dis 16: 383-386.

Li Y, Wang J, Zhu X, Feng Q, Li X, Feng X (2012) Urinary protein markers predict the severity of renal histological lesions in children with mesangial proliferative glomerulonephritis. BMC Nephrol 13: 29.

Li Y, Yuan T, Lu W, Chen M, Cheng X, Deng S (2012) Association of tuberculosis and polymorphisms in the promoter region of macrophage migration inhibitory factor (MIF) in a Southwestern China Han population. Cytokine 60: 64-67.

Liu R, Xu N, Wang X, Shen L, Zhao G, Zhang H, Fan W (2012) Influence of MIF, CD40, and CD226 polymorphisms on risk of rheumatoid arthritis. Mol Biol Rep 39: 6915-6922.

Lolis E (2011) Glucocorticoid counter regulation: macrophage migration inhibitory factor as a target for drug discovery. Curr Opin Pharmacol 1: 662-668.

Matsumoto K, Maruyama N, Maruyama T, Ohnishi Y, Nonaka S, Inoshita A, Ito K, Kitajima S, Abe M, Satomura A, Fujita T (2005) Elevated macrophage migration inhibitory factor (MIF) levels in the urine of patients with focal glomerular sclerosis. Clin Exp Immunol 139: $338-347$.

Mehls O, Hoyer PF (2011) Dosing of glucocorticosteroids in nephrotic syndrome. Pediatr Nephrol 26: 2095-2098.

Renner P, Roger T, Calandra T (2005) Macrophage migration inhibitory factor: gene polymorphisms and susceptibility to inflammatory diseases. Clin Infect Dis 41: 513-519.
Shiroeda H, Tahara T, Nakamura M, Shibata T, Nomura T, Yamada H, Hayashi R, Saito T, Yamada M, Fukuyama T, Otsuka T, Yano H, Ozaki K, Tsuchishima M, Tsutsumi M, Arisawa T (2010) Association between functional promoter polymorphisms of macrophage migration inhibitory factor (MIF) gene and ulcerative colitis in Japan. Cytokine 51: 173-177.

Smoak KA, Cidlowski JA (2004) Mechanisms of glucocorticoid receptor signaling during inflammation. Mech Ageing Dev 125: 697-706.

Sreih A, Ezzeddine R, Leng L, LaChance A, Yu G, Mizue Y, Subrahmanyan L, Pons-Estel BA, Abelson AK, Gunnarsson I, Svenungsson E, Cavett J, Glenn S, Zhang L, Montgomery R, Perl A, Salmon J, Alarcón-Riquelme ME, Harley JB, Bucala R (2011) Dual effect of the macrophage migration inhibitory factor gene on the development and severity of human systemic lupus erythematosus. Arthritis Rheum 63: 3942-3951.

Stahn C, Buttgereit F (2008) Genomic and nongenomic effects of glucocorticoids. Nat Clin Pract Rheumatol 4: 525-533.

Stosic-Grujicic S, Stojanovic I, Nicoletti F (2009) MIF in autoimmunity and novel therapeutic approaches. Autoimmun Rev 8: 244-249.

Van Husen M, Kemper MJ (2011) New therapies in steroid-sensitive and steroid-resistant idiopathic nephrotic syndrome. Pediatr Nephrol 26: 881-892.

Vivarelli M, D’Urbano LE, Stringini G, Ghiggeri GM, Caridi G, Donn R, Tozzi A, Emma F, De Benedetti F (2008) Association of the macrophage migration inhibitory factor $-173^{*} \mathrm{C}$ allele with childhood nephrotic syndrome. Pediatr Nephrol 23: 743-748.

Xie Q, Wang SC, Bian G, Zhan FL, Xie JK, Li J (2012) Association of MIF-173G/C and MBL2 codon 54 gene polymorphisms with rheumatoid arthritis: A meta-analysis. Hum Immunol 73: 966-971.

Zheng X, Wang D, Hou S, Zhang C, Lei B, Xiao X, Kijlstra A, Yang P (2012) Association of macrophage migration inhibitory factor gene polymorphisms with Behçet's disease in a han chinese population. Ophthalmology 119: 2514-2518. 